\title{
Urolithiasis in adults with congenital megaureter
}

\author{
Gregory S. Rosenblatt, MD; Ken Takesita, MD; Gerhard J. Fuchs, MD, FACS
}

\begin{abstract}
The primary presentation of congenital megaureter in adults is rare. Development of urolithiasis may lead to this unusual underlying diagnosis. Urinary tract stones can form either within the dilated ureteral segment or in a part of the upper urinary tract proximal to the abnormal ureteral segment. We report two cases of nephrolithiasis that occurred in adults found to have segmental megaureter. The first case is that of a 58-year-old man who presented with left lower quadrant pain. Computed tomography scan revealed a $2-\mathrm{cm}$ stone in the distal left ureter within an area of isolated segmental distal ureteral dilation. The second case is a 48-year-old man who developed recurrent renal urolithiasis associated with isolated distal megaureter.

Although a rare condition in adults, congenital megaureter may present when kidney stones develop as a result of the ureteral abnormality. Typically, stones will develop within the dilated segment of ureter. Atypically, stones may develop away from the site of the underlying abnormality. Congenital megaureter is a diagnosis that urologists and radiologists need to consider in the setting of isolated distal ureteral dilation, as the diagnosis of adult megaureter may require more involved surgical measures to prevent recurrence of adverse symptoms.
\end{abstract}

Can Urol Assoc J 2009;3(6):E77-E80

\section{Introduction}

Initially described by Caulk in 1923, congenital megaureter is a condition usually diagnosed in neonates and children; its primary presentation in adults is rare. ${ }^{1}$ Spontaneous resolution occurs in over half of all affected children, and this is hypothesized to occur as a result of the ureterovesical junction (UVJ) maturation and growth. ${ }^{2-4}$ Diagnostic criteria include: dilated ureter, absence of vesicoureteral reflux, absence of infravesical obstruction and absence of distal ureteral obstruction. ${ }^{5}$ When this condition does present in adults, it typically does so during the third or fourth decade, and unilateral disease (most often left-sided) is more common than bilateral disease. ${ }^{6-7}$ Herein we present two unusual cases of patients with urolithiasis associated with unilateral isolated distal megeureter.
Between July 1, 2007, and December 31, 2008, two cases of nephrolithiasis presented in patients with evidence of primary segmental ureteral dilation.

\section{Case 1}

A 58-year-old man presented with isolated left lower quadrant pain. He had spontaneously passed uric acid stones on two prior occasions, but he had not required urologic surgery. Computed tomography (CT) scan revealed a $2.3-\mathrm{cm}$ stone in a dilated segment of his left distal ureter (Fig. 1a). Retrograde ureteropyelogram was performed at the time of retrograde stone treatment, which revealed a dilated distal left ureter with a normal-sized proximal ureter and minimal dilation of the ipsilateral collecting system (Fig. 1b). Direct visualization of the stone and ureter at the time of retrograde ureteroscopy showed the stone to be contained within a dilated ureteral segment with a normal-calibre proximal ureter (Fig. 2). Ureteroscopic intervention was not noted to be especially difficult, and no additional dilation of the ureteral orifice was required. The stone was fragmented using Holmium laser technology, and all fragments were removed using a stone basket. A ureteral stent was left in place for one week, and the patient had no subsequent episodes of pain. At the 8-week follow-up, renal ultrasonography showed physiologic distention of the renal collecting system, similar to what was seen prior to surgery.

\section{Case 2}

A 48-year-old man presented with left-side flank pain. Renal ultrasound showed a 9-mm non-obstructing calculus in the left renal pelvis with minimal hydronephrosis and no renal parenchymal thinning. He did not have a history of fever, and there was no other indication of urinary tract infection. There was also no prior history of urinary infections, and he denied a history of difficult urination. Urine dipstick analysis revealed trace blood without evidence of leukocyte esterase or nitrite. His serum creatinine was $1.0 \mathrm{mg} / \mathrm{dL}$ (normal: 0.8 to $1.5 \mathrm{mg} / \mathrm{dL}$ ).

He had undergone retrograde intrarenal surgery about 5 years before for the management of left renal stone 


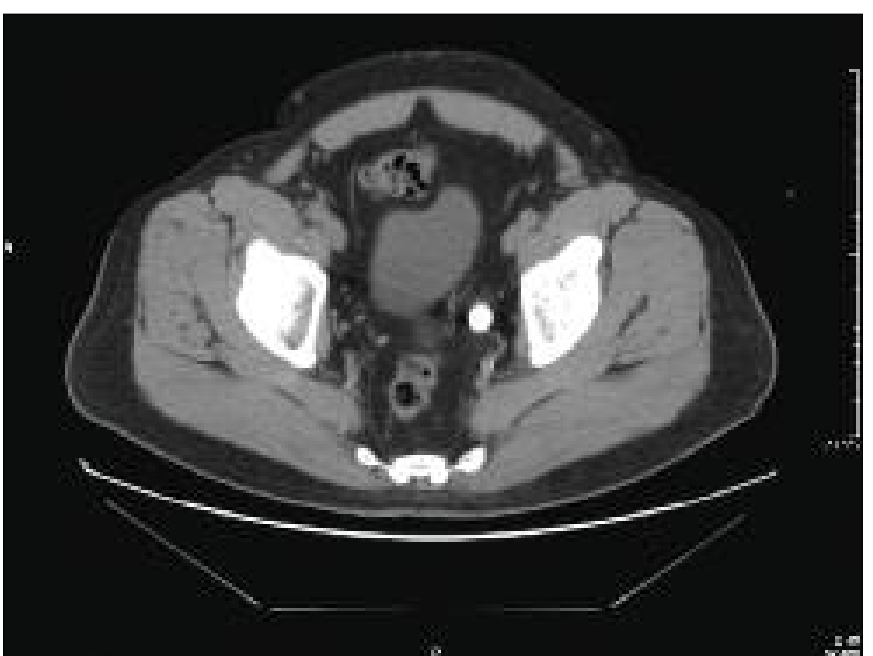

Fig. 1a. Computed tomography scan depicting a $2.3-\mathrm{cm}$ distal left ureteral stone.

disease. His other medical history included hypertension, gout and obesity treated by gastric bypass. Computed tomography scan without intravenous contrast was performed around the time of his prior stone treatment, and this showed isolated distal left ureteral dilation (Fig. 3a) and normalappearing left renal parenchyma without significant dilation of the left renal collecting system (Fig. 3b).

Cystourethroscopy, retrograde ureterorenoscopy and retrograde intra-renal surgery were performed. A retrograde ureteropyelogram showed significant distal left ureteral dilation (Fig. 4a) and mild dilation of the left renal collecting system (Fig. 4b). Ureteroscopy confirmed that there was no physical obstruction of the distal ureter and UVJ. The distal ureter was voluminous immediately above the intramural segment, and proximally it tapered to normal calibre. Under direct vision, the kidney stone was fragmented using Holmium laser technology, and the fragments were extracted using a flat-wire basket. A ureteral stent was left
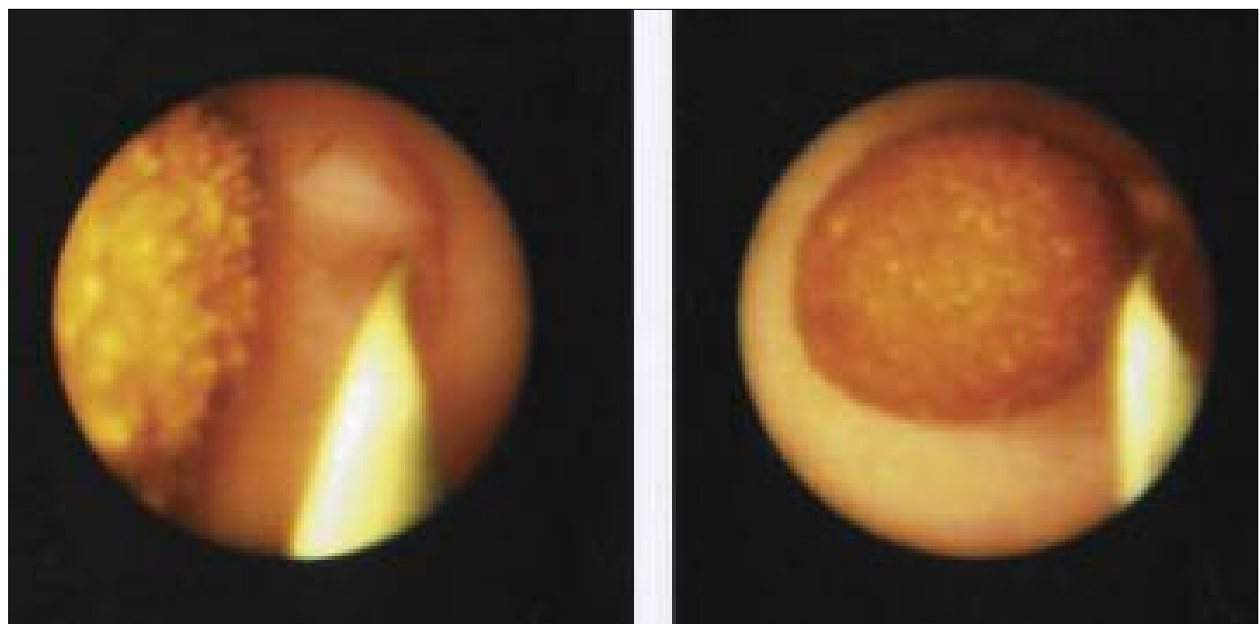

Fig. 2. Ureteroscopic view of a large, 2.3-cm stone that formed within the dilated distal ureteral segment. Of note is the narrowing to normal calibre proximally.

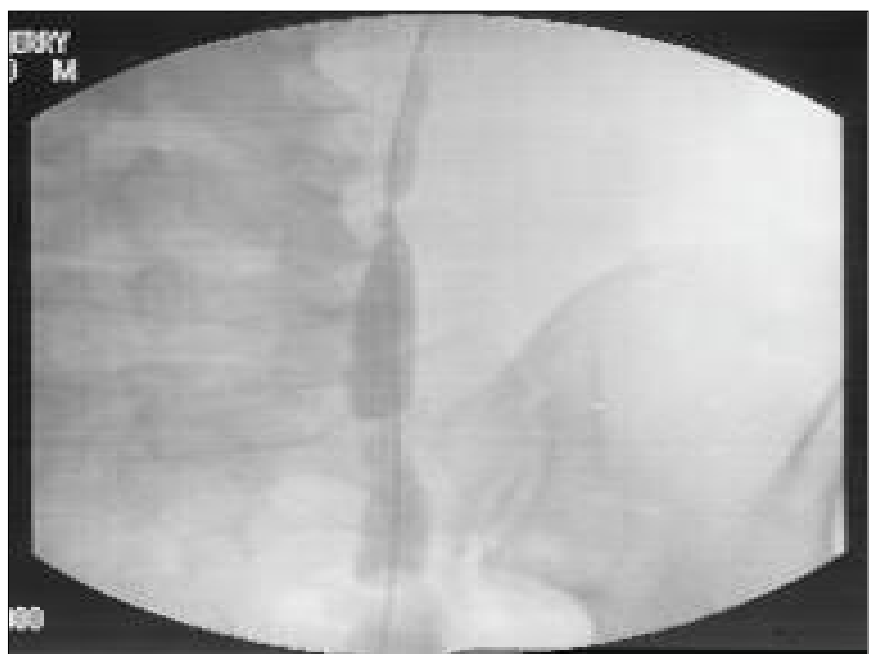

Fig. 1b. Retrograde ureterogram that shows distal ureteral dilation and normal-calibre ureter proximally.

for one week, and there was no operative or perioperative complication.

\section{Discussion}

The current belief is that primary obstructive megaureter presents primarily in adults when the congenital abnormality does not cause symptoms or illness and is not seen via an imaging study performed as children. Spontaneous regression fails to occur, yet patients remain asymptomatic through childhood and into their adult years. Eventual symptoms that may occur include: urinary tract infections, renal parenchymal damage and recurrent stone formation.

In the largest reported series of 55 adults and adolescents with symptomatic primary obstructive megaureter, Hemal and colleagues identified 20 patients (36\%) as having urinary tract calculi. ${ }^{8}$ Most of the calculi were located in the ureter; only 3 of the 55 (5\%) patients had renal calculi without calcification near the location of the underlying abnormality (distal ureter). Large stones can develop in the dilated portion of the ureter due to stasis of urine. Delakas and colleagues described an adult patient with primary megaureter who developed a $12-\mathrm{cm}$ urinary calcification within the dilated portion of the ureter. ${ }^{9}$

Case 2 is unusual in that the patient developed renal calculi without ureteral calculi in the presence of an ipsilateral distal ureteral megaureter. The retrograde ureterogram depicts the isolated distal ureteral dilatation, as does the $\mathrm{CT}$ scan. The 


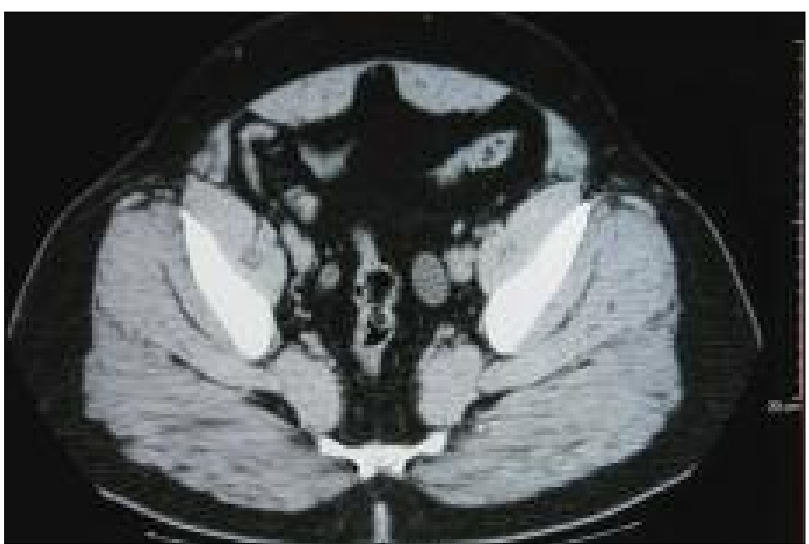

Fig. 3a. Computed tomography depicting distal left ureteral dilation.

marked dilation in the distal ureter without comparable dilation in the more proximal portion of the urinary tract suggests a functional rather than physical obstruction. Further imaging would be required to confirm persistent dilation of the distal ureter since completing surgical treatment of the stone. Our best estimate is that the distal ureter remains dilated, as there was no physical obstruction, and there has been no intervention to correct the underlying abnormality.

The exact underlying cause of megaureter is not known. Hypotheses of ureteral wall muscle-fibre abnormalities and abnormal collagen composition/deposition have been explored. ${ }^{10-13}$ When we assessed the retrograde ureterogram in this patient, we were struck by the stark difference between the amount of distal ureteral dilation and the relative minimal dilation of the more proximal ipsilateral urinary tract. Stone development in this patient may have occurred secondary to urinary stasis that was transmitted to the upper tract. However, the stasis that is transmitted to the upper tract does not appear to be severe enough to cause thinning of the renal parenchyma. He had developed stones in that kidney on two occa-

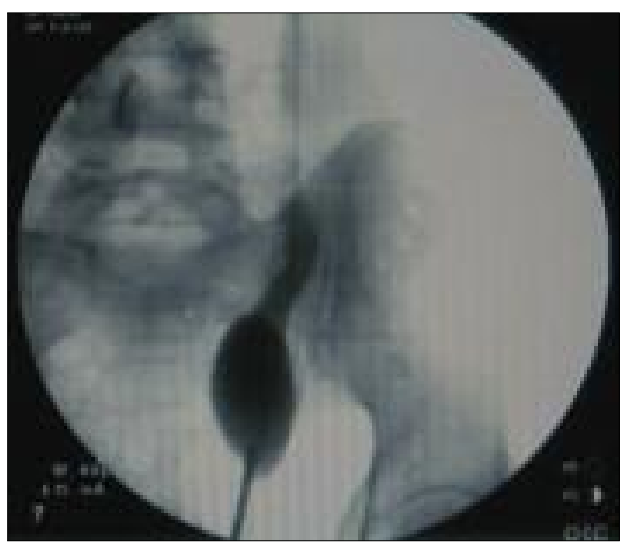

Fig. 4a. Retrograde left-side ureteropyelogram confirming isolated distal ureteral.

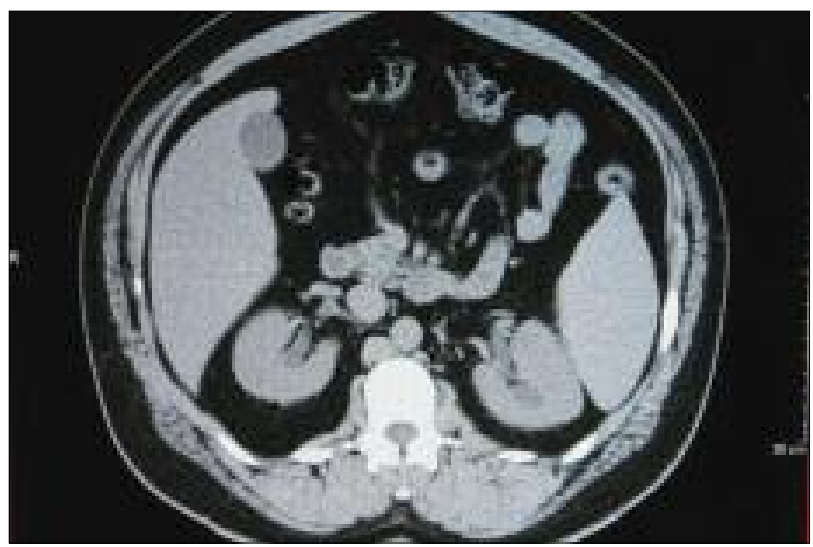

Fig. 3b. More proximal upper urinary tract without significant dilation.

sions necessitating surgical intervention: the first was a mix of uric acid $(60 \%)$ and calcium oxalate $(40 \%)$; the second stone was calcium oxalate. Metabolic workup may help to identify additional factors contributing to stone formation, but this has not yet been performed.

We feel that continued hydration and medical management with regular monitoring are appropriate in both patients. Should persistent and recurrent urolithiasis become a chronic problem, another option for surgical management has been discussed with these patients, which would involve excision of the abnormal distal ureter and unilateral ureteroneocystostomy.

From the Minimally Invasive Urology Institute, Cedars-Sinai Medical Center, Los Angeles, CA

\section{Competing interests: None declared.}

This paper has been peer-reviewed.

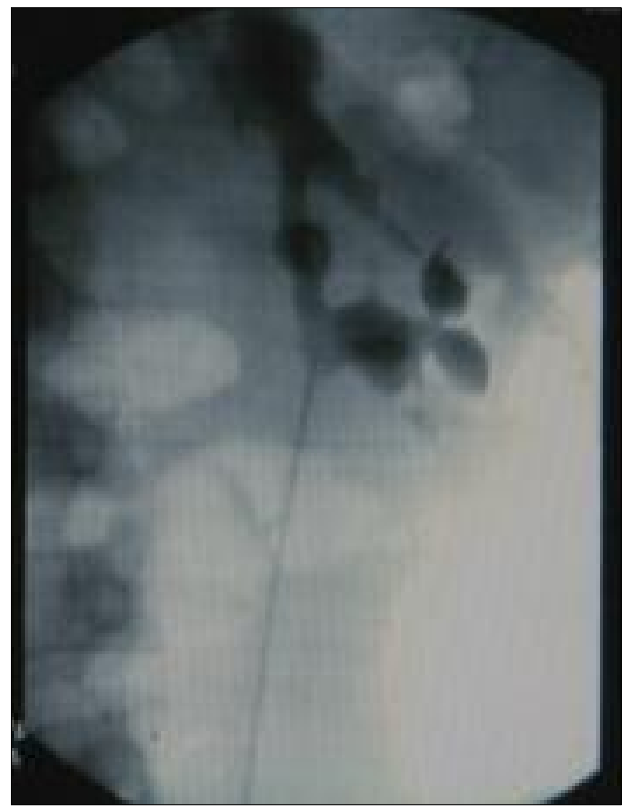

Fig. 4b. The proximal left upper urinary tract and collecting system is minimally dilated, compared to the gross segmental dilation of the distal ipsilateral ureter. 


\section{References}

1. Caulk JR. Megaloureter: the importance of the ureterovesical valve. J Urol 1923; 9:315-9.

2. Pitts WR Jr, and Muecke EC. Congenital megaloureter: a review of 80 patients. J Urol 1975; 111:46873.

3. Oliveira EA, Diniz JS, Rabelo EA, et al. Primary megaureter detected by prenatal ultrasonography: conservative management and prolongad follow-up. Int Urol Nephrol 2000;32:13-8.

4. Domini M, Aquino A, Pappalepore N, et al. Conservative treatment of neonatal primary megaureter. Eur J Pediatr Surg 1999;9:396-9.

5. Khoury A, Bagli DJ. Reflux and Megaureter. In: Wein AJ, Kavoussi LR, Novick AC, Partin AW, Peters CA, eds. Campbell-Walsh Urology. $9^{\text {th }}$ ed. Philadelphia: Elsevier Inc; 2007:3467-81.

6. Dorairajan LN, Hemal AK, Gupta NP, et al. Primary obstructive megaureter in adults: need for an aggressive management strategy. Int Urol Nephrol 1999;31:633-41.

7. Hanna MK, Wyatt JK. Primary obstructive megaureter in adults. J Urol 1975;113:328-35.

8. Hemal AK, Ansari MS, Doddamani D, et al. Symptomatic and complicated adult and adolescent primary obstructive megaureter - indications for surgery: analysis, outcome, and follow-up. Urology 2003;61: 703-7.
9. Delakas D, Daskalopoulos G, Karyotis I, et al. Giant ureteral stone in association with primary megaureter presenting as an acute abdomen. Eur $J$ Rad 2002;41:170-2.

10. Mackinnon KJ, Foote JW, Wiglesworth FW, et al. The pathology of the adynamic distal ureteral segment. J Urol 1970;103:134-7.

11. Gosling JA, Dixon JS. Functional obstruction of the ureter and renal pelvis. A histological and electron microscopic study. Br J Urol 1978;50:145-52.

12. Notley RG. Electron microscopy of the primary obstructive megaureter. Br J Urol 1972;44:229-34.

13. Hanna MK, Jeffs RD, Sturgess JM, et al. ureteral structure and ultrastructure. Part II. Congenital ureteropelvic junction obstruction and primary obstructive megaureter. J Urol 1976;116:725-30.

Correspondence: Dr. Gregory S. Rosenblatt, Minimally Invasive Urology Institute at Cedars-Sinai Medical Center, 8635 W. $3^{\text {rd }}$ Street, \#1070W, Los Angeles, CA 90048; fax: 310-423-4711; grrosen@aol.com 